LIAMES 6 - pp. 7-28, Primavera 2006

\author{
Kristine Stenzel ${ }^{1}$ \\ (Musen Nacional/ UFRJ)
}

\title{
As categorias de evidencialidade em Wanano (Tukano Oriental)
}

\begin{abstract}
This paper describes the complex and typologically interesting evidential system in Wanano, one of the sixteen languages of the Eastern Tukanoan family spoken in northwest Amazonia. Wanano verb morphology is quite complex and includes obligatory verb-final marking of clause modality, organized into four major categories by which statements, questions, and 'oriented' utterances are grammatically marked. The most complex sub-system of clause modality is the evidential system, which marks (primarily) realis statements. The paper explores the core semantics-linked to source of evidence-of each of the five evidential categories: HEARSAY (RELATADA), VISUAL, NON (NÃO)-VISUAL, INFERENCE (INFERÊNCIA), and ASSERTION (SuPosição). It also explores instances which show the extended use of evidentials to code other types of situations as well as areas of semantic overlap. It also outlines questions related to the relationship between evidentials and epistemic values, and to the relationship between evidentiality and the notions of realis/irrealis. It shows that Wanano evidential categories display sensitivity to scalar values of speaker commitment and that certain evidential categories span the frontier between the spheres of realis/irrealis.
\end{abstract}

KEYWORDS: Eastern Tukanoan languages; Evidentiality; Typology of evidential systems; Epistemic modality

RESUMO: Este artigo descreve o sistema de marcação da evidencialidade, complexo e tipologicamente interessante, do Wanano, uma das dezesseis línguas da família Tukano Oriental, faladas no noroeste amazônico. A morfologia verbal em Wanano é bastante rica e inclui marcação obrigatória de modalidade oracional, organizada em quatro categorias principais pelas quais afirmações, perguntas e falas 'orientadas' são gramaticalmente indicadas. O subsistema de modalidade oracional mais complexo é o sistema de evidencialidade, que marca (primariamente) afirmações no modo realis. Minha análise explora a semântica básica - baseada em fonte de informação/evidência - das cinco categorias de evidencialidade: RELATADA, VISUAL, NÃO-VISUAL, INFERÊNCIA e SUPOSIÇÃo. Exemplifica, também, as extensões semânticas de algumas categorias a outras situações e aponta áreas de sobreposição semântica. Coloca questões ligadas à relação entre marcação de evidencialidade e a expressão de valores epistêmicos, bem como à relação entre a marcação de evidencialidade e os conceitos de realis/irrealis. Mostra que as categorias de evidencialidade em Wanano exprimem valores epistêmicos e que uma das categorias ocupa um território de fronteira entre os domínios do realis e do irrealis.

PALAVRAS-CHAVE: Família lingüística Tukano Oriental; Evidencialidade; Tipologia de sistemas de evidenciais; Modalidade epistêmica.

${ }^{1}$ Pesquisadora PD do CNPq. A pesquisa das línguas Wanano e Piratapuyo recebeu apoio financeiro do Endangered Languages Fund, da Wenner-Gren Foundation for Anthropological Research, da National Science Foundation (Grant 0211206), e da National Science Foundation/National Endowment for the Humanities Documenting Endangered Languages Program (FA-52150-05), bem como apoio institucional e logístico do Instituto Socioambiental e do Museu Nacional/Universidade Federal do Rio de Janeiro. 


\section{INTRODUÇÃO}

A evidencialidade é uma categoria gramatical que indica a fonte de informação na qual o falante baseia uma afirmação (Chafe e Nichols, 1986; Aikhenvald, 2003). Tipologicamente, a evidencialidade é uma categoria relativamente rara e que tende a ocorrer como traço areal em algumas poucas regiões geográficas ${ }^{2}$. A Amazônia é uma região de grande concentração de línguas com sistemas de evidencialidade, que são encontrados em línguas das famílias Arawá, Arawak, Carib, Chibcha, Nambiquara, Pano, Quechua, Tukano, Tupí-Guaraní, Vaupés-Japurá (Makú), Witoto, Yanomami e Záparo, além de línguas isoladas como Andoke e Paez (Aikhenvald e Dixon, 1998; Epps, 2005; Landaburu, 2005). Dessas, as línguas da família Tukano, e especialmente as do ramo oriental, exibem sistemas de marcação de evidencialidade dos mais complexos do mundo.

As dezesseis línguas Tukano-Oriental (TO) são faladas por aproximadamente 28.000 pessoas no noroeste amazônico, na região fronteiriça entre o estado brasileiro do Amazonas e o distrito colombiano do Vaupés ${ }^{3}$. Nessas línguas, a evidencialidade é marcada obrigatoriamente em todas as frases do tipo indicativo e os paradigmas de evidencialidade são compostos por quatro ou cinco categorias. Geralmente, a evidencialidade é marcada no verbo através de um sufixo verbal portmanteau que codifica, além da fonte de informação, categorias gramaticais de pessoa, gênero, número e tempo/aspecto. No entanto, há categorias de evidencialidade que em algumas línguas TO são expressas por construções analíticas com verbos auxiliares, como veremos mais adiante.

Neste trabalho, descrevo o sistema de marcação de evidencialidade em Wanano, língua TO falado por aproximadamente 1.500 pessoas. Na seção 2, faço um resumo da morfologia do verbo finito em Wanano e discuto o papel dos evidenciais na marcação da modalidade oracional. Na seção 3 analiso as funções e as formas das cinco categorias de evidencialidade, RELATADA, VISUAL, NAO-VISUAL, INFERÊNCIA e SUPOSIÇÃO, oferecendo exemplos de algumas das suas extensões semânticas. Na seção 4 aponto algumas questões teóricas importantes relacionadas ao estudo de sistemas de evidencialidade e sugiro metas para investigações futuras.

\footnotetext{
${ }^{2}$ Fora das Américas, onde se concentram as línguas com sistemas de evidencialidade, tais sistemas são encontrados em Japonês, na região dos Bálcãs (em Búlgaro, Macedônio, e Albanês), no Cáucaso (em Turco), e em línguas da região do Himalaia (Sherpa, Akha e Tibetano).

${ }^{3}$ As línguas TO faladas no Brasil e na Colômbia são Desano, Kubeo, Waikhana (Piratapuyo), Tukano, Tuyuca, Siriano e Wanano. As línguas Bará, Barasana, Karapana, Makuna, Pisamira, Retuarã, Taiwano, Tatuyo e Yuruti são faladas somente na Colômbia.
} 


\section{APALAVRA VERBALEM WANANO}

TABELA 1. A morfologia do verbo finito

\begin{tabular}{|c|c|c|c|c|c|c|c|c|}
\hline \multicolumn{8}{|l|}{ RADICAL } & \\
\hline \multirow[t]{2}{*}{ SIMPLES } & \multicolumn{7}{|c|}{$\begin{array}{l}\text { COMPOSTO } \\
\text { (Raízes dependentes) }\end{array}$} & \\
\hline & +1 & +2 & +3 & +4 & +5 & +6 & +7 & +8 \\
\hline Raiz & $\begin{array}{l}\text { ASPECTO } \\
\text { (DIR., } \\
\text { MAN., } \\
\text { ETC) }\end{array}$ & $\begin{array}{c}\text { ASPECTO } \\
\text { (COMPL., } \\
\text { INCOA., } \\
\text { ETC) }\end{array}$ & $\begin{array}{l}\text { MODALI- } \\
\text { DADE } \\
\text { (DEÔNTI- } \\
\text { CA) }\end{array}$ & $\begin{array}{l}\text { NEGA- } \\
\text { ÇÃO }\end{array}$ & $\begin{array}{l}\text { INTENSI- } \\
\text { FICAÇÃO }\end{array}$ & $\begin{array}{l}\text { MODALI- } \\
\text { DADE } \\
\text { (DESID., } \\
\text { DUB., } \\
\text { ETC) }\end{array}$ & $\begin{array}{c}\text { ASPECTO } \\
\text { (PERF/ } \\
\text { IMPERF) }\end{array}$ & $\begin{array}{c}\text { MODALIDADE } \\
\text { ORACIONAL } \\
\text { EVIDENCIAIS } \\
\text { IMPERATIVOS } \\
\text { INTERROGATIVOS } \\
\text { IRREALIS }\end{array}$ \\
\hline
\end{tabular}

(O sombreamento indica constituintes obrigatórios)

Na Tabela 1 vemos a ordem básica e a categorização dos morfemas que ocorrem regularmente em verbos finitos em Wanano ${ }^{4}$. A palavra verbal é minimamente composta por dois constituintes: uma raiz verbal e um marcador de modalidade oracional (representados nas colunas sombreadas na Tabela 1). Mas a palavra verbal pode ser morfologicamente muito mais complexa, composta por várias raízes formando um radical complexo, e afixado por uma série de morfemas opcionais. A primeira coluna à esquerda representa a raiz verbal principal, a cabeça fonológica e morfológica da palavra verbal. As colunas de +1 a +3 representam as categorias de raízes verbais que ocorrem mais freqüentemente em radicais compostos. As raízes que ocorrem nas posições +1 e +2 são, geralmente, verbos de movimento que codificam dois tipos de informação: adverbial, indicando nuances da realização espacial de uma ação, i.é. movimento circular, cislocativo, translocativo (as raízes sito e ta em 1a) ou aspectual, indicando alguma qualidade interna da ação, i.é. realização completa, incoativo, à distância (ruka em 1b). Na posição +3 ocorrem raízes de processos mentais. Radicais compostos com essas raízes codificam modalidade deôntica: indicam a habilidade ou a necessidade do falante de realizar a ação do verbo principal ( basi 'saber' em 1c)
(1) a. to-i
du'te - sito - ta - a.
$\sim$ da-ta-a
REM-LOC cortar-MOV.circular-vir-SUP:PERF
carregar-vir-SUP:PERF
'Foi em volta cortando (folhas). Trouxe de volta'

\footnotetext{
${ }^{4}$ Para uma análise detalhada da morfologia verbal em Wanano, ver capítulos 5 e 6 de (Stenzel, 2004).

${ }^{5}$ A nasalização tanto em Wanano com em outras línguas TO é morfêmica, e é indicada nos exemplos por ' ' antes do morfema. Nesses morfemas, todas as vogais, obstruintes sonoras e aproximantes são realizadas como alofones nasalizados. Assim, em (1), a realização dos morfemas nasalizados é da [nã],

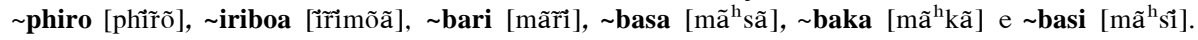


b. phiro - ro - we'rt ti - ro - re chowe - ruka - a cobra-SG-AUMEN ANAF-SG-OBJ vomit-INCOA-SUP:PERF

'A cobra grande começou a vomitar (o cachorro)'
c. $\sim$ iriboa - re $\sim$ bari $\sim$ basa do'a - ti - ra
limão-OBJ 1PL.INCL pessoa doença-VBZ-VIS:IMPERF.2/3

\author{
baka - basi - ka \\ procurar-DEON-SUP:IMPERF \\ 'Quando adoecemos, devemos procurar limão'
}

Imediamente após o radical simples (uma raiz) ou complexo (duas ou mais raízes), ocorrem morfemas opcionais que codificam negação (2a), intensificação (2b), e outros tipos de modalidade que expressam a atitude do falante perante a ação do verbo principal, i.é. dúvida, desejo, frustração, etc. (2c) e aspecto perfectivo/imperfectivo (2d). A coluna mais à direita $(+8)$ representa a categoria de modalidade oracional.

(2)

$\begin{array}{lll}\text { a basa cht- era - ka } & \text { die - ro - re } \\ \text { pessoa comer-NEG-SUP:IMPERF } & \text { cachorro-SG-OBJ } \\ \text { 'Gente não come cachorro' } & \end{array}$

b. ti-ro kta - yłidt- a - wa'a - a

ANAF-SG estar.com.medo-INTENS-AFF-ficar-SUP:PERF

'Ficou muito amedrontado'

c. to $-\mathrm{pu} \quad \sim \mathrm{st} \quad \sim \mathrm{yt} \quad \sim$ baka - ba - a

REM-LOC chegar ver procurar-FRUST-SUP:PERF

'Chegou lá, olhou, procurou (em vão)'.

d. ytü-se'e bu'e - wa'a yoa - ati - i

1SG - CONTR. estudar - ir fazer - IMPERF-VIS.PERF. 1

'Eu, por outro lado, estava sempre indo embora para estudar'

A Tabela 2 complementa a Tabela 1 e mostra o paradigma completo das categorias de modalidade oracional que compõe a última posição morfológica da palavra verbal. 
STENZEL: As CATEGORIAS DE EVIDENCIALIDADE EM WANANO

TABELA 2. Morfologia do verbo finito: modalidade oracional

\begin{tabular}{|c|c|c|c|c|}
\hline RADICAL & \multicolumn{4}{|c|}{$\stackrel{+8}{\text { MODALIDADE ORACIONAL }}$} \\
\hline \multirow{5}{*}{$\begin{array}{c}\text { RAIZ + } \\
\text { morfemas } \\
\text { opcionais } \\
\text { nas } \\
\text { posições } \\
+1 \mathrm{a}+7\end{array}$} & \multicolumn{3}{|c|}{ REALIS $<------------>$ IRREALIS } & \\
\hline & \multicolumn{3}{|c|}{ AFIRMAÇÕES } & \\
\hline & \begin{tabular}{|cc}
\multicolumn{2}{|c}{ (EVIDÊNCIAS) } \\
VISUAL & NÃO-VISUAL \\
INTERFERÊNCIA & SUPOSIÇÃO \\
RELATADA &
\end{tabular} & \multicolumn{2}{|c|}{$\begin{array}{cc}\text { PREDIÇÃO } & \text { INTENÇÃO } \\
\text { ESPECULAÇÃO } & \text { NEGAÇÃO }\end{array}$} & $\begin{array}{l}\text { 'ORIENTADA' } \\
\text { IMPERATIVO } \\
\text { PERMISSIVO } \\
\text { ADVERSÃO } \\
\text { ADMONIÇÃO }\end{array}$ \\
\hline & \multicolumn{3}{|c|}{ INTERROGATIVAS } & \\
\hline & $\begin{array}{l}\text { IMPERFECTIVO PERFECTIVO } \\
\text { SUPOSTO }\end{array}$ & ESPECUL & ATIVO & \\
\hline
\end{tabular}

A modalidade oracional é marcada obrigatoriamente em todo verbo finito e os morfemas dessa categoria são mutuamente exclusivos. Cada oração é marcada como a) uma afirmação realis, por uma das categorias de evidencialidade; b) uma afirmação irrealis: predição, especulação, intenção; c) uma pergunta, com nuances de evidencialidade; ou, d) uma fala orientada a outros: imperativo, permissivo. O subsistema mais complexo de modalidade oracional é o da evidencialidade, que marca afirmações realis, o equivalente em Wanano de uma frase indicativa básica.

É importante ressaltar que a função dos evidenciais e dos outros marcadores de modalidade oracional não é de dar informação sobre a natureza interna do evento expresso pelo verbo. A informação relativa ao evento em si é codificada pelas categorias da morfologia verbal apresentadas na Tabela 1. A morfologia final do verbo, por outro lado, codifica a relação do falante com o evento ou situação através de atos de fala - ele afirma ou questiona algo relacionado ao evento, ou ele orienta uma fala relacionada ao evento para um interlocutor. Nesse esquema, a função dos evidenciais é de indicar a informação acerca da relação cognitiva entre o falante e o evento, a perspectiva do falante, mediada por uma fonte de informação, sobre o evento. 


\section{ASCATEGORIAS DE EVIDENCIALIDADE EMWANANO}

TABELa 3. Categorias de Evidencialidade

\begin{tabular}{|c|c|c|c|c|c|c|c|c|c|}
\hline \multirow{3}{*}{\multicolumn{2}{|c|}{ DE OUTROS }} & \multicolumn{8}{|c|}{ PRIMEIRA MÃO } \\
\hline & & \multicolumn{6}{|c|}{ EXTERNA } & \multirow{2}{*}{\multicolumn{2}{|c|}{ INTERNA }} \\
\hline & & \multicolumn{5}{|c|}{ DIRETA } & INDIRETA & & \\
\hline \multicolumn{2}{|c|}{ RELATADA } & \multicolumn{4}{|c|}{ VISUAL } & NÃO-VISUAL & INFERÊNCIA & \multicolumn{2}{|c|}{ SUPOSIÇÃO } \\
\hline ESP. & DIF. & \multicolumn{2}{|c|}{$1^{\mathrm{a}}$} & \multicolumn{2}{|c|}{$2^{a} / 3^{a}$} & \multirow{3}{*}{ koa(-ta)- } & \multirow{3}{*}{-ri hi- } & & \\
\hline \multirow{2}{*}{-yu’ka } & \multirow{2}{*}{-yu’ti } & PERF & IMPF & PERF & IMPF & & & PERF & IMPF \\
\hline & & $-\mathrm{i}$ & -ha & -re & $-\mathrm{ra}$ & & & $-\mathrm{a}$ & $-\mathrm{ka}$ \\
\hline
\end{tabular}

Vemos na Tabela 3 as categorias de evidencialidade em Wanano. A parte superior da tabela é uma matriz de traços que caracterizam cinco categorias de evidência: RELATADA, VISUAL, NÃO-VISUAL, INFERÊNCIA, e SUPOSIÇÃo. Cada nível da matriz representa uma dicotomia fundamental na caracterização da evidência ou da fonte de informação. No nível mais alto, por exemplo, há uma oposição entre informação cuja fonte, de alguma forma, é o próprio falante, informação de 'primeira-mão', e informação que provém 'de outros', uma dicotomia em si suficiente para caracterizar e estabelecer a categoria de (informação) RELATADA. Para caracterizar as quatro categorias restantes, que compartilham o traço primeira-mão, há dicotomias adicionais que estabelecem distinções entre fontes de informação externa/interna, direta/indireta, e visual/não-visual ${ }^{6}$.

As formas sincrônicas dos evidenciais estão na parte inferior da tabela; há uma qualificação adicional interna para algumas categorias (i.é. diferenciação aspectual nas categorias VISUAL e SUPOSIÇÃo). Notamos também algumas diferenças de forma: as categorias NÃO-VISUAL e INFERÊNCIA são codificadas por construções analíticas ao invés de sufixos.

Chamo atenção para o fato de que a minha análise do sistema evidencial em Wanano difere de análises anteriores (Waltz e Waltz, 1997; 2000) em dois aspectos significativos. Em primeiro lugar, às quatro categorias identificadas por Waltz e Waltz - RELATADA, VISUAL, INFERÊNCIA e SUPOSIÇÃO - acrescento mais uma: a categoria de evidência NÃO-VISUAL ${ }^{7}$. Em segundo lugar, mostrarei que as distinções internas às categorias de evidencialidade não se

${ }^{6}$ Considero a oposição 'primeira-mão'/‘outros' como primária, como Barnes (1984) e Malone (1988), mas de fato, há várias maneiras de organizar os traços definidores dos sistemas evidenciais em línguas TO, cada uma com resultado interessante. Por exemplo, na sua análise do Tatuyo e do Barasana, Gomez-Imbert (1999) coloca a distinção direta/indireta como primária e, assim, estabelece uma dicotomia inicial entre as categorias sensoriais diretas e as categorias indiretas de INFERÊNCIA e RELATADA. Essa opção resulta numa organização do sistema que reflete a tipologia de sistemas de evidencialidade proposta por Willett (1988).

${ }^{7}$ A categoria rotulada como Não-visual nas análises de Waltz e Waltz corresponde à categoria de suPosição em minha análise. 
baseiam em 'tempo', como analisam Waltz e Waltz, e sim, em distinções de aspecto e referência.

\subsection{Informação de outros: a categoria 'RELATADA'}

A categoria de evidência RELATADA se distingue pelo fato da fonte de informação não ser o próprio falante e, sim, terceiros. Em outras palavras, ao marcar uma frase com um evidencial da categoria RELATADA, o falante sinaliza que a informação transmitida se originou de alguma outra pessoa, como nas frases em (1).
(3) a. ti - ro wt' $t-p t$
wa'a- yu'ka
ANAF-SG casa-LOC
ir - REP.ESP

(Alguém me contou que) 'Ele foi para casa'

$\begin{array}{lll}\text { b. ti - ro } & \text { wt' } t-p t & \text { wa'a-yu'ti } \\ \text { ANAF-SG } & \text { casa - LOC } & \text { ir - REP.DIFF }\end{array}$

(Dizem que) 'Ele foi para casa'

As formas -yu'ka e -yu'ti expressam distinções de referência, ou seja, distinções entre tipos de 'terceiros' que são as fontes originais da informação (ver também Floyd 1999:130-140). O falante de Wanano marca uma afirmação com -yu'ka para indicar que ainda tem em mente a fonte original da informação, o seja, o referente específico que forneceu a informação. Ele usa esse sufixo, por exemplo, ao relatar algo que acaba de ouvir, provavelmente de uma pessoa diretamente envolvida no evento. Frases marcadas por -yu'ti, por outro lado, indicam a impossibilidade de recuperar ou identificar a fonte original da informação, ou seja, remetem a um referente difuso. Usa-se esse sufixo, por exemplo, para relatar fofocas ou notícias que chegam de longe ${ }^{8}$.

\subsection{Evidência primeira-mão e externa: as categorias VISUAL, NÃO-VISUAL, e INFERÊNCIA}

Das quatro categorias evidenciais marcadas pelo traço primeira-mão na Tabela 3, três delas também indicam informação provinda de fontes externas: as categorias VISUAL, NÃO-VISUAL e INFERÊNCIA. As categorias externas ainda se subdividem por uma distinção entre fontes de informação direta e indireta. As categorias de fonte direta são VISUAL e NÃO-VISUAL, ambas indicando que o falante tem conhecimento do evento através de algum tipo de evidência sensorial direta.

${ }^{8}$ Devo dizer, no entanto, que essa análise se baseia em dados elicitados, pois frases espontâneas com o evidencial RELATADA ocorrem raramente. Na fala do dia-a-dia, há uma clara preferência por citar diretamente frases de outros. Aikhenvald (2002:190) nota essa mesma tendência em Tariana e de forma generaliza em toda a região do Vaupés. 
3.2.1. A categoria vISUAL

\begin{tabular}{|c|c|c|c|c|c|c|c|c|c|}
\hline \multicolumn{2}{|c|}{ DE OUTROS } & \multicolumn{8}{|c|}{ PRIMEIRA MÃO } \\
\hline & & \multicolumn{6}{|c|}{ EXTERNA } & \multirow{2}{*}{\multicolumn{2}{|c|}{ INTERNA }} \\
\hline & & \multicolumn{5}{|c|}{ DIRETA } & INDIRETA & & \\
\hline \multicolumn{2}{|c|}{ RELATADA } & \multicolumn{4}{|c|}{ VISUAL } & NÃO-VISUAL & INFERÊNCIA & \multicolumn{2}{|c|}{ SUPOSIÇÃO } \\
\hline ESP. & DIF. & \multicolumn{2}{|c|}{$1^{\mathrm{a}}$} & \multicolumn{2}{|c|}{$2^{a} / 3^{a}$} & \multirow{3}{*}{ koa(-ta)- } & \multirow{3}{*}{-ri hi- } & & \\
\hline \multirow{2}{*}{-yu`ka } & \multirow{2}{*}{-yu’t } & PERF & IMPH & PERF & IMPF & & & PERF & IMPF \\
\hline & & $-\mathrm{i}$ & -ha & -re & - ra & & & $-a$ & $-\mathrm{ka}$ \\
\hline
\end{tabular}

Frases que levam sufixos da categoria de evidência visual são as mais comuns na fala do dia-a-dia. A sua semântica básica indica que o falante afirma algo do seu conhecimento direto ou da sua experiência própria. A categoria visual é ao mesmo tempo a mais básica e a mais complexa do sistema, pois o paradigma de seus sufixos demonstra um maior refinamento semântico: inclui uma distinção de pessoa (primeira pessoa / 'o resto' $)^{9}$ e uma distinção de aspecto (imperfectivo / perfectivo). Vemos exemplos com os sufixos de primeira pessoa em (4) e exemplos com os sufixos de segunda/terceira pessoa em (5).

(4) a. $y t^{\prime}$

$$
\text { hi - ha }
$$

ko - iro

$1 \mathrm{sG}$

COP-VIS:IMPERF. 1

parente-NOM:SG

'Sou (seu) parente'

b. $\sim \mathrm{bu}$ ' $\mathrm{H}$

cht - dua - re

2SG

comer-DESID-OBJ

'Trouxemos o que você queria comer'
(5) a. $\sim \mathrm{o}-\mathrm{pt}$
hi - ra
yt
$\sim$ pho'da
DEIC: PROX-LOC
COP-VIS:IMPERF. 2/3
1sG.POSS
filhos

'(Ele, o cachorro) está aqui, meus filhos'

${ }^{9} \mathrm{O}$ paradigma de marcação $1^{\mathrm{a}} / 2^{\mathrm{a}}-3^{\mathrm{a}}$ pessoa é uma inovação encontrada apenas nas línguas Wanano e Waikhana (Piratapuyo) e o seu desenvolvimento diacrônico ainda é um mistério. Em todas as outras línguas da família TO, o paradigma se organiza por uma oposição $3^{\mathrm{a}} / 1^{\mathrm{a}}-2^{\mathrm{a}}$ pessoa, certamente o padrão mais antigo. Esse padrão de fato ainda é encontrado no paradigma Wanano de marcação de frases irrealis e em nominalizações. 
StenZel: As CATEGORIAS DE EVIDENCIALIDADE EM WANANO

\author{
b. $\mathrm{bt}-\mathrm{kt}-\sim \mathrm{da}$ \\ khta - ati - re st'o - pt-ka \\ vê velho-MASC-PL \\ ter-IMPERF-VIS:PERF. $2 / 3$ \\ peneirar-CLS:cesto- \\ st'o - puka \\ CLS:arredondado \\ 'Nossos antepassados tinham (esse tipo de) peneira'
}

Aspecto ou tempo? Vale a pena nos deter um pouco na questão da diferença entre distinções de tempo e de aspecto nas línguas TO. De fato, 'tempo' aparece como elemento fundamental dos sistemas evidenciais em grande parte da literatura sobre línguas TO (Barnes, 1984; Morse e Maxwell, 1999; Miller, 1999; Jones e Jones, 1991; Waltz e Waltz, 1997, 2000; Ramirez, 1997). Porém, o conceito de tempo aparece altamente qualificado, pois a definição mais tradicional - de que tempo marca um evento como passado, presente ou futuro em relação ao momento da fala (Givón, 2001:285-6) - não se encaixa bem na estrutura semântica dessas línguas. Nas línguas TO, a categoria de tempo é sempre subordinada às várias categorias de evidência e a sua interação com essas categorias produz alguns resultados semânticos inesperados. Barnes (1984:265) nota, por exemplo, que em Tuyuca, nas categorias de INFERÊNCIA e SUPOSIÇÃO ${ }^{10}$, distinções de tempo indicam "quando o evento ocorreu" (uma leitura coerente com a definição tradicional), mas nas categorias VISUAL, NÃO-VISUAL e RELATADA, as distinções de tempo indicam "quando o falante obteve a informação"l1.

Outros autores (Gomez-Imbert, 1999, 2000; Morse e Maxwell, 1999; Ferguson et al., 2000; Stenzel, 2004) analisam a diferenciação interna dos sufixos da categoria visUAL como expressão de valores aspectuais - perfectivo (marcando um evento ou estado como tendo limites, pontos de início e/ou fim) e imperfectivo (evocando a noção do 'sem limites', marcando um evento ainda não terminado ou um estado que ainda vigora) - e não valores temporais. Partindo desse pressuposto e lembrando que os sufixos finais do verbo em Wanano codificam a natureza da relação do falante com a sua fala, podemos esperar que frases com formas imperfectivas impliquem, na perspectiva do falante, um acesso ilimitado ou ainda vigente à fonte de informação e frases com formas perfectivas impliquem (de novo, da perspectiva do falante) uma fonte de informação bloqueada, inacessível ou terminada.

É claro que nem sempre é fácil identificar essas diferenças, pois tempo e aspecto especialmente tempo passado e aspecto perfectivo — estão fortemente associados e, de fato, se sobrepõem semanticamente com certa freqüência, como em (4) e (5) acima. Mas todo cuidado é pouco na hora de analisar exemplos como esses, principalmente se nós nos atemos apenas às glosas em inglês ou português, pois essas sugerem que estamos diante de distinções de tempo. É necessário procurar e reconhecer casos em que a sobreposição semântica de tempo/aspecto não ocorre.

\footnotetext{
10 'APPARENT' e 'ASSUMED' no texto de Barnes.

${ }^{11}$ Nas análises de Waltz e Waltz de Wanano, porém, não há nenhuma qualificação dessa natureza. Presume-se que adotam uma definição tradicional para a categoria de tempo.
} 
Consideremos os enunciados em (6), retirados de uma narrativa em que um $\sim$ wati 'diabo da floresta' ${ }^{12}$ gruda numa mulher e a carrega até a sua casa, num pau oco no meio do mato. Os filhos da mulher, vendo o rapto da mãe e a entrada do 'diabo' no pau oco, correm para encontrar o pai e o levam à casa do malvado, na floresta. Ao chegar, o homem pergunta pela sua esposa (6a) e os filhos respondem que ela está dentro do pau oco (6b).

\begin{tabular}{|c|c|c|c|}
\hline (6) a. & $\begin{array}{l}\sim \text { do'o - i } \\
\text { INT - LOC }\end{array}$ & $\begin{array}{l}\text { hi - ri } \\
\text { COP-INT }\end{array}$ & $\begin{array}{l}\sim \text { di - a } \\
\text { dizer-SUP:PERF }\end{array}$ \\
\hline & ‘'Onde ela está?’ & perguntou (o pai) & \\
\hline b. & $\begin{array}{l}\sim 0 \text { - i } \\
\text { DEIC:PROX-LOC }\end{array}$ & $\begin{array}{l}\text { hi - re } \\
\text { COP-VIS:PERF. } 2 / 3\end{array}$ & $\begin{array}{l}\sim \text { di - a } \\
\text { dizer-SUP:PERF }\end{array}$ \\
\hline
\end{tabular}

Chamo atenção à resposta dos filhos. Eles viram a mãe sendo levada para dentro do pau oco e estão afirmando que é lá que ela ainda se encontra; então, porque não usam a forma presente ou imperfectiva do evidencial VISUAL? A resposta é que mesmo que ainda vigore o estado 'mãe dentro-do-pau-oco', o acesso visual dos filhos a essa informação já cessou. É essa relação (de limite entre o falante e a sua fonte de informação) que está sendo codificado pela forma perfectiva do sufixo evidencial.

Em (7), de uma outra narrativa de $\sim$ wati, um grupo de tais criaturas chega numa casa com o objetivo de comer seus habitantes: uma mulher e os seus filhos. A mulher logo desconfia do plano e inventa uma desculpa para fugir com seus filhos até o rio. Os $\sim$ wati correm atrás dela e os enunciados em (7) ocorrem no momento em que eles chegam à beira e vêem que a mulher conseguiu escapar, nadando embora com os filhos. Mesmo que a mulher já tenha fugido de fato, não ocorre a forma passada ou perfectiva do evidencial na fala do 'diabo'. A explicação dessa escolha é simples: ele opta pela forma imperfectiva porque a sua fala se baseia numa fonte de informação que continua diante dos seus olhos_ele ainda vê a mulher nadando no rio.
a. $\sim$ waku- basi - ko
$\sim$ bu' $) \sim$ ya - ka
du'ti - ra
pensar-saber-FEM
2SG ser.mal-ENF
fugir-VIS:IMPERF. 2/3

'Conseguiu fugir, mulher espertinha'
b. bu't-re
cht- doka - bo - a
$2 \mathrm{SG}-\mathrm{OBJ}$
comer-COMPL-DUB-SUP:PERF

'Nos íamos comê-la todinha'

Esses exemplos confirmam que as distinções aspectuais desses sufixos de evidencialidade não se referem à natureza do evento ou ao estado expresso pelo predicado,

\footnotetext{
${ }^{12}$ São entes perversos e perigosos que vivem na mata, mas que podem tomar forma humana e que têm poderes ou características mágicas. São de natureza diferente de outros seres mágicos como o Curupira (ver Jackson, 1983:207-208).
} 
mas à relação entre o falante e a fonte de evidência. No mais, mostram que a despeito de (muitos) exemplos em que tempo e aspecto se sobrepõem, uma análise que priorize distinções aspectuais é certamente mais apropriada. As formas imperfectivas da categoria VISUAL indicam a proximidade cognitiva do falante ao evento ou estado, graças a uma evidência ainda accessível, enquanto as formas perfectivas indicam uma distância cognitiva entre o falante e o evento, graças a uma evidência não accessível. Situações assim codificadas se referem, frequientemente, a eventos de fato ocorridos no passado, como em (5), e, possivelmente, derivam de distinções temporais outrora ativas e que se gramaticalizaram (ver discussões em Bybee et al., 1994 e de Haan, 2001a). É bem possível que distinções de tempo sejam um traço gramatical diacrônico da família TO, já que elas ainda se manifestam em muitas línguas. Sincronicamente, porém, em línguas como o Wanano, são distinções aspectuais e não temporais o traço saliente nos marcadores de evidencialidade.

Extensões semânticas da categoria de evidência viSUAL. Evidenciais da categoria VISUAL ocorrem tanto com verbos de estado quanto com verbos ativos, como vimos nos exemplos acima, mas há certas combinações com a forma imperfectiva que resultam em leituras semânticas específicas. Quando os sufixos imperfectivos da categoria VISUAL ocorrem com verbos ativos, eles indicam ações habituais vivenciadas ou presenciadas pelo falante (8). Os sufixos imperfectivos afixados a verbos de estado têm a função de expressar fatos ou conhecimentos gerais (9). Extensões semânticas dessa natureza, em que são empregados marcadores da categoria VISUAL na expressão de fatos gerais e de ações habituais, têm sido freqüentemente notadas em sistemas de evidencialidade complexas (Aikhenvald, 2003:13).
(8) a. $\sim \mathrm{de}$ to - pt-re ti - pt-re tidi - era - ha
NEG REM-LOC-OBJ ANAF-LOC-OBJ andar/caçar-NEG-VIS:IMPERF.1
'Nunca vou caçar naquele lugar'

$\begin{array}{lrl}\text { b. wt'a } & \text { soa wipe } & \text { yoa - ra } \\ \text { descascar } & \text { ralar tipicar fazer-VIS:IMPERF.2/3 }\end{array}$

'(As mulheres wanano) descascam, ralam, e espremem (a mandioca no tipiti)'.
(9) a. a'ri - ro hi - ra ba'a
DEM:PROX-SG COP-VIS:IMPERF.2/3 tucunaré

'Este é tucunaré'
b. $\sim$ phuri - a
veneno-NOM ter-NOM-SG
khta - ri - ro hi - ra
COP-VIS:IMPERF. 2/3
ti - ro
'Ele (a cobra) é venenosa'
ANAF-SG 
3.2.2. A categoria NÃO-VISUAL

\begin{tabular}{|c|c|c|c|c|c|c|c|c|c|}
\hline \multicolumn{2}{|c|}{ DE OUTROS } & \multicolumn{8}{|c|}{ PRIMEIRA MÃO } \\
\hline & & \multicolumn{6}{|c|}{ EXTERNA } & \multirow{2}{*}{\multicolumn{2}{|c|}{ INTERNA }} \\
\hline & & \multicolumn{5}{|c|}{ DIRETA } & INDIRETA & & \\
\hline \multicolumn{2}{|c|}{ RELATADA } & \multicolumn{4}{|c|}{ VISUAL } & NÃO-VISUAL & INFERÊNCIA & \multicolumn{2}{|c|}{ SUPOSIÇÃO } \\
\hline ESP. & DIF. & \multicolumn{2}{|c|}{$1^{\mathrm{a}}$} & \multicolumn{2}{|c|}{$2^{a} / 3^{a}$} & \multirow{3}{*}{ koa(-ta)- } & \multirow{3}{*}{-ri hi- } & & \\
\hline \multirow{2}{*}{-yu’ka } & \multirow{2}{*}{-yu’ti } & PERF & IMPF & PERF & IMPF & & & PERF & IMPF \\
\hline & & $-\mathrm{i}$ & -ha & -re & $-\mathrm{ra}$ & & & $-\mathrm{a}$ & $-k a$ \\
\hline
\end{tabular}

A segunda categoria externa e direta de evidência primeira-mão é a categoria NÃO-VISUAL, utilizada em afirmações baseadas em percepção sensorial não-visual. O uso mais freqüente dessa categoria indica evidência auditiva, como em (10a), onde a falante ouve a chegada de um grupo de homens vindo para raptá-la, e (10b), onde o falante ouve, de dentro de um abrigo, que um Curupira acaba de cair no chão, morto, do lado de fora.

(10) a. dubi-a ya'a- ida ta-a $\sim$ di - a koa-ta - ra mulher-PL pegar-NOM:PL vir-NOM PROG-NOM NÃO.VIS-vir-VIS:IMPERF.2/3 'Seqüestradores de mulheres estão chegando'. (Estou ouvindo)

$\begin{array}{lcl}\text { b. bora- } \sim \mathrm{su}-\mathrm{ka} & \text { wa'a - ro } & \text { koa - ta - a } \\ \text { cair - COMPL-ENF } & \text { ir - NOM } & \text { NÃO.VIS-vir-SUP:PERF } \\ \text { '(Ele) caiu'. (ouvi a sua caída) } & \end{array}$

No entanto, a categoria NÃO-VISUAL também pode ser usada para indicar outros tipos de percepção sensorial, como em (11), onde o falante descreve o gosto da carne de vaca.
to
di'i $\sim$ doa - ro
koa - ka
3sG.POSS carne ser.bom-NOM:ADV
NÃO.VIS-SUP:IMPERF
'A sua carne (da vaca) é gostosa'

Vemos nesses exemplos que a evidência NÃO-VISUAL é indicada por uma construção analítica contendo um verbo auxiliar. Essa construção consiste sempre do verbo koa, que significa algo como 'perceber por meio sensorial', e, no caso da audição, o verbo dependente ta 'vir', indicando que a evidência sensorial auditiva sempre chega de fora (enquanto a degustação é uma percepção intrinsecamente interior, indicada somente por koa). 
O radical verbal com koa (simples ou composto) leva um sufixo de uma das duas categorias principais de evidência primeira-mão: VISUAL ou SUPOSIÇÃo. VISUAL é a categoria 'default' indicando evidência direta: -re (perfectiva) ou -ra (imperfectiva), como em (10a). Sufixos da categoria de suPosição ocorrem em duas instâncias. A forma perfectiva -a é usada em narrativas (10b), enquanto a forma imperfectiva -ka é usada para indicar as sensações internas do falante, como em (11). O verbo que precede a construção NÃO-VISUAL é sempre nominalizado.

Não devemos estranhar a ocorrência de um marcador evidencial viSUAL numa construção evidencial NÃO-VISUAL: apesar dos rótulos, não há contradição semântica. Estudos comparativos mostram que a categoria VISUAL tende a ser a menos marcada formalmente e, em muitos casos, chega a ter realização Ø (Aikhenvald, 2004a:10). Em sistemas com categorias sensoriais múltiplas, como o de Wanano, visUAL pode ser considerado a categoria geral não-marcada de evidência sensorial direta. Assim, quando sufixos da categoria VISUAL marcam o verbo diretamente, eles indicam, por default, evidência visual. Quando ocorrem como constituintes de uma construção analítica como a NÃO-VISUAL em Wanano, eles indicam apenas evidência sensorial direta (perfectiva ou imperfectiva).

Os exemplos acima mostram que a categoria NÃO-VISUAL ocupa um espaço semântico próprio dentro do sistema de evidencialidade em Wanano. Semanticamente, as categorias VISUAL e NÃO-VISUAL são contrastivas, essa última sendo a categoria de evidência sensorial marcada.

\subsubsection{INFERÊNCIA}

\begin{tabular}{|c|c|c|c|c|c|c|c|c|c|}
\hline \multicolumn{2}{|c|}{ DE OUTROS } & \multicolumn{8}{|c|}{ PRIMEIRA MÃO } \\
\hline & & \multicolumn{6}{|c|}{ EXTERNA } & \multirow{2}{*}{\multicolumn{2}{|c|}{ INTERNA }} \\
\hline & & \multicolumn{5}{|c|}{ DIRETA } & INDIRETA & & \\
\hline \multicolumn{2}{|c|}{ RELATADA } & \multicolumn{4}{|c|}{ VISUAL } & NÃO-VISUAL & INFERÊNCIA & \multicolumn{2}{|c|}{ SUPOSIÇÃO } \\
\hline ESP. & DIF. & \multicolumn{2}{|c|}{$1^{\mathrm{a}}$} & \multicolumn{2}{|c|}{$2^{\mathrm{a} / 3^{\mathrm{a}}}$} & \multirow{3}{*}{ koa(-ta)- } & \multirow{3}{*}{-ri hi- } & & \\
\hline \multirow{2}{*}{-yu’ka } & \multirow{2}{*}{-yu’ti } & \multirow{2}{*}{ PERF } & \multirow{2}{*}{ IMPF } & \multirow{2}{*}{ PERF } & \multirow{2}{*}{ IMPF } & & & PERF & IMPF \\
\hline & & & & & & & & $-a$ & $-\mathrm{ka}$ \\
\hline
\end{tabular}

INFERÊNCIA é a terceira categoria de evidência primeira-mão no paradigma Wanano. Essa categoria compartilha o traço externa com as categorias VISUAL e NÃO-VISUAL, e se distingue dessas pelo traço indireto. Como a categoria NÃO-VISUAL, INFERÊNCIA é indicada por uma construção analítica com um verbo auxiliar, com nominalização da raiz que antecede o sufixo -ri. O verbo auxiliar nessa construção é a cópula hi, marcado (como no caso da construção NÃO-VISUAL) por um sufixo ou da categoria VISUAL ou de SUPOSIÇÃo, sendo os sufixos da categoria VISUAL -re (perfectivo) e -ra (imperfectivo) os mais comuns na fala do dia-a-dia. 
Frases que apresentam essa construção indicam que o falante apresenta sua conclusão sobre um evento ou estado baseado em algum indicador ou resultado observado. É essa observação por parte do falante que qualifica a INFERÊNCIA como categoria de evidência primeira-mão. No entanto, como toda conclusão desse tipo é baseada em observação de resultados, e não na observação da ocorrência em si, trata-se de evidência indireta.

Consideramos o enunciado em (12), de uma narrativa em que um grupo de homens chega numa maloca distante com plano de raptar mulheres para desposar. Mas, ao entrar na maloca, encontram somente redes desocupadas e concluem:
yoa - ta - pu wiha - tu'st- ri
hi - ra
ser.longe-REF-LOC MOV.p/fora-COMPL.REC- NOM.INFER
COP-VIS:IMPERF. $2 / 3$
'Jáfugiram'

Em (13), um wati cujo filho saiu para caçar e nunca mais voltou conclui dessa ausência que:
(13)

$\begin{array}{llll}\mathrm{yu} & \sim \mathrm{ba}-\mathrm{ku}-\mathrm{re} & \sim \text { waha }- \text { roka }-\mathbf{r i} & \text { hi }- \text { re } \\ \text { 1SG.POSS } & \text { filho-MASC-OBJ } & \text { matar-DIST-NOM.INFER } & \text { COP-VIS:PERF.2/3 } \\ \text { 'Meu filho foi morto' } & & \end{array}$

Como no caso da construção NÃO-VISUAL, a construção que marca INFERÊNCIA pode levar sufixos da categoria VISUAL, como em (12) e (13), ambos exemplos de falas de protagonistas de narrativas. Mas a forma perfectiva da categoria suPosição é o marcador default das falas do narrador de mitos e outras textos não pessoais, como vemos em (13). A fala ocorre num momento da narrativa em que um homem retorna, após quatro anos, ao lugar em que teve um encontro com um Curupira, que acabou morrendo (ver 9b). Ele não encontra mais restos do corpo do Curupira e o narrador conclui o óbvio:
ba - yu'du-ka
wa'a - ri
hi - a
decompor-INTENS-AFF ir-NOM.INFER
'Tinha decomposto completamente'
COP-SUP:PERF

\subsubsection{Evidência primeira-mão e interna: SUPOSIÇão}

\begin{tabular}{|c|c|c|c|c|c|c|c|c|c|}
\hline \multicolumn{2}{|c|}{ DE OUTROS } & \multicolumn{8}{|c|}{ PRIMEIRA MÃO } \\
\hline & & \multicolumn{6}{|c|}{ EXTERNA } & \multirow{2}{*}{\multicolumn{2}{|c|}{ INTERNA }} \\
\hline & & \multicolumn{5}{|c|}{ DIRETA } & INDIRETA & & \\
\hline \multicolumn{2}{|c|}{ RELATADA } & \multicolumn{4}{|c|}{ VISUAL } & NÃO-VISUAL & INFERÊNCIA & \multicolumn{2}{|c|}{ SUPOSIÇÃO } \\
\hline ESP. & DIF. & \multicolumn{2}{|c|}{$1^{\mathrm{a}}$} & \multicolumn{2}{|c|}{$2^{\mathrm{a} / 3^{\mathrm{a}}}$} & \multirow{3}{*}{ koa(-ta)- } & \multirow{3}{*}{-ri hi- } & & \\
\hline \multirow{2}{*}{-yu’ka } & \multirow{2}{*}{-yu`ti } & PERF & IMPF & PERF & IMPF & & & PERF & IMPF \\
\hline & & $-\mathrm{i}$ & -ha & -re & -ra & & & $-\mathrm{a}$ & $-\mathrm{ka}$ \\
\hline
\end{tabular}


A quinta categoria do sistema de marcação de evidencialidade é suPosição, cuja semântica é particularmente interessante. É uma categoria extremamente rara, pois frases marcadas com sufixos dessa categoria indicam que o falante baseia sua afirmação não em evidência externamente acessível, mas em evidência interna ou internalizada. Tal evidência pode se fundamentar tanto em experiência prévia do próprio falante - resultando em suposições lógicas - quanto em seus conhecimentos culturais, históricos ou físicos do mundo - resultando em afirmações de fato. Como na categoria visual, há formas perfectivas e imperfectivas da categoria SUPOSIÇão, mas nessa categoria não há diferenciação de pessoa.

Já vimos que a forma perfectiva da categoria SUPOSIÇÃo -a é o sufixo default em mitos e narrativas não-pessoais. Ocorre em todas as frases em que o falante exerce papel de narrador, como em (10b) e (14) acima, e em (15), as linhas introdutórias da narrativa do Curupira. Nessas linhas introdutórias e em todas as frases do falante como narrador ocorre o sufixo perfectivo da categoria de suPOSIÇÃo (em negrito em 15a e 15c). Nas falas em (15b) e (15c), no entanto, ocorre também a fala direta do protagonista principal do texto (sublinhado). Fala direta (ou seja, a fala de qualquer protagonista) em narrativas é como toda fala do dia-a-dia: pode levar evidenciais de qualquer categoria.

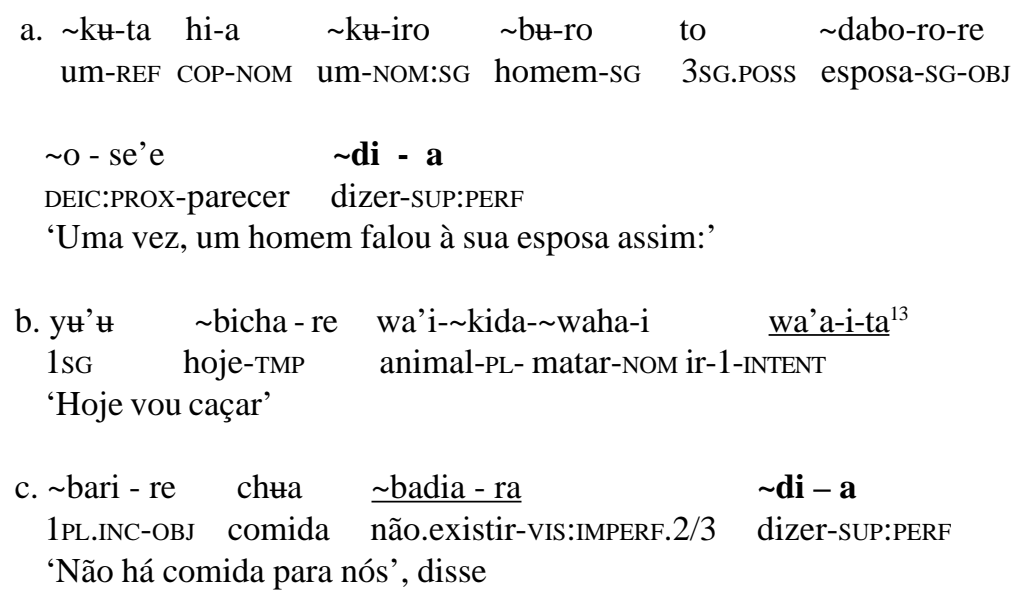

A forma imperfectiva da categoria suPosição é semanticamente multifuncional, mas podemos identificar um elo comum entre todos os seus usos: são todas afirmações que se baseiam em evidência interna ou internalizada. Em primeiro lugar, o falante de Wanano usa a forma imperfectiva -ka na expressão de suas emoções (16a), sensações corporais (16b), processos cognitivos (16c) e habilidades (16d). Em outras palavras, -ka marca experiências internas e sentimentos - tipos de informação afirmadas pelo falante na ausência de evidência externa.

${ }^{13} \mathrm{O}$ sufixo -ta pertence à categoria de marcadores de irrealis, expressando a intenção do falante de realizar alguma atividade no futuro. Para uma análise completa de todos os sufixos da categoria de modalidade oracional, ver capítulo 7 de (Stenzel, 2004). 


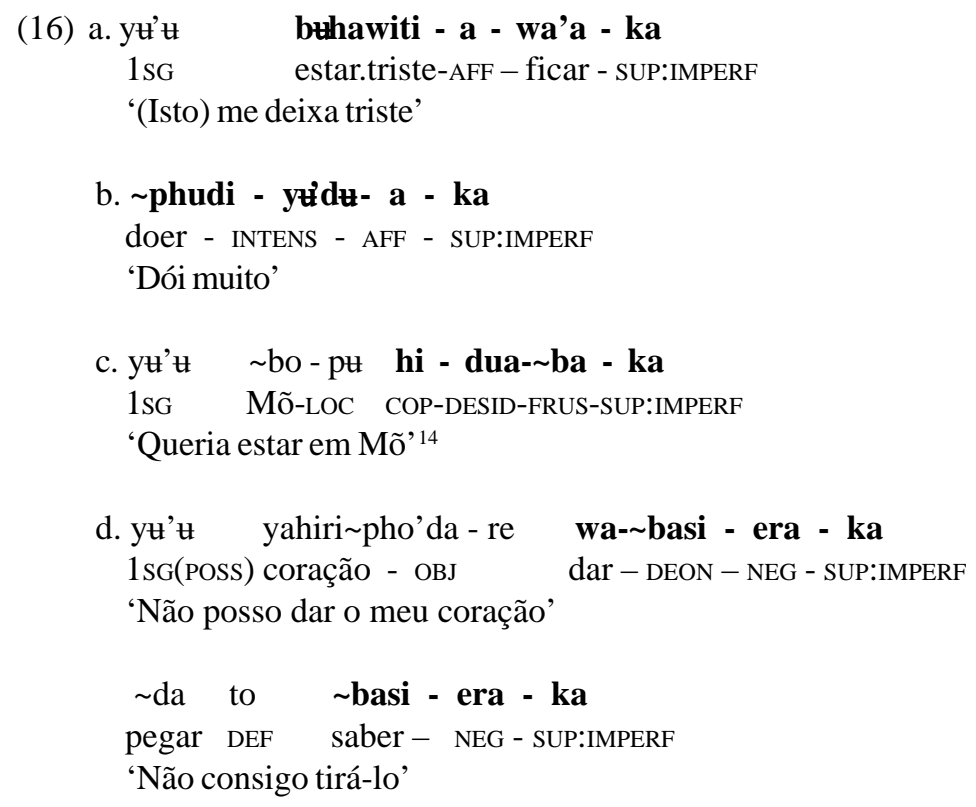

O segundo uso do imperfectivo -ka é na expressão de suposições lógicas relativas aos estados ou ações de outros. O falante não precisa de evidência externa para tais afirmações - o seu conhecimento das pessoas envolvidas, as suas experiências anteriores em situações semelhantes ou do mundo em geral constituem base suficiente.

Voltamos à narrativa do encontro do homem com o Curupira. Antes da fala em (17) o Curupira pedira educadamente o coração do homem, mas este, sabendo como são maliciosos os Curupiras, supõe que suas intenções são verdadeiras:

$\begin{array}{llll}\text { yu' } t r e & \text { a'ri - ro } & \text { cht-dua - ro } & \sim \text { di }- \text { ka } \\ \text { 1SG - OBJ } & \text { DEM:PROX-SG } & \text { comer-DESID-NOM } & \text { PROG-SUP:IMPERF } \\ \text { 'Esse (o Curupira) está querendo me comer' } & \end{array}$

Uma pequena conversa que presenciei demonstra, também, o uso do evidencial -ka numa suposição lógica. Fui com um amigo à casa do irmão deste, Eugênio, por volta das 13 horas. Logo antes de chegar, encontramos o vizinho de Eugênio e perguntamos por ele. Mesmo não o tendo visto naquele dia, o vizinho conhecia os hábitos do Eugênio o suficiente para supor que ele estaria em casa na hora do almoço.
Meu amigo: Eugênio
hi - hari
Eugênio
COP-INT.IMPERF
'Eugênio está?'

\author{
O vizinho: hi - ka \\ COP-SUP:IMPERF \\ Está'
}

\footnotetext{
${ }^{14}$ Moa poaye, nome da comunidade de Caruru Cachoeira, em Wanano.
} 
O terceiro uso da forma imperfectiva -ka se dá na expressão dos conhecimentos internalizados do falante, conhecimentos gerais adquiridos como parte de sua herança cultural e histórica. Vemos nessa função uma sobreposição das esferas semânticas dos imperfectivos das categorias SUPOSIÇÃo e VISUAL, na expressão de fatos. Veja-se o exemplo em (19), retirado de um texto escrito por uma professora Wanano.

O texto fala do macaco guariba e há um desenho de macaco que acompanha a historinha. A primeira linha do texto introduz o animal e nessa linha a autora usa o sufixo da categoria VISUAL no verbo cópula hi justamente porque o leitor vê o macaco no desenho. Nas outras linhas, a forma imperfectiva da categoria SUPOSIÇão -ka marca cada afirmação sobre os hábitos dessa espécie de macaco. A autora não precisa necessariamente ter visto um macaco guariba realizando as atividades descritas no texto para poder afirmá-las como fatos, pois conhecer os hábitos de animais da floresta faz parte do seu conhecimento geral do mundo.

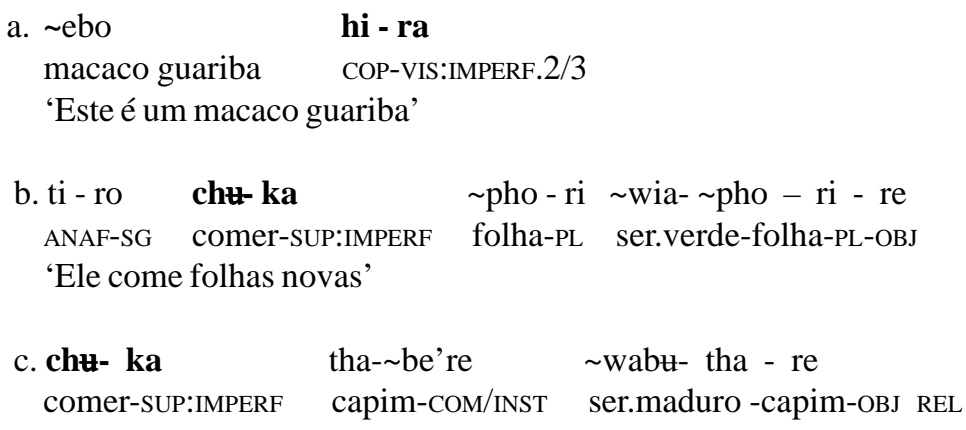

Em (20) temos um exemplo raro de uma fala caracterizada, aparentemente, por uma dupla marcação de evidencialidade, já que apresenta a construção de INFERÊNCIA, -ri hi-, e o sufixo imperfectivo de suPosição, -ka. Necessitamos de informação contextual para a análise deste exemplo, tirado da mesma narrativa do encontro entre o homem e o Curupira. A fala em (20) ocorre tarde na narrativa, no momento em que o homem retorna ao lugar do encontro, onde o corpo do Curupira havia caído e se decomposto (ver exemplo 14). Ao mexer na terra com seu terçado, o homem faz reviver o Curupira, que levanta de seu sono profundo (morte) e declara: 
(20) $y t^{\prime}$ t $~$ khari - yt'du-a

wa'a - ri

hi - ka

$1 \mathrm{SG}$ dormir-INTENS -AFF ir-NOM.INFER

COP-SUP:IMPERF

'Fiquei adormecido por muito tempo'

Há de fato, aqui, duas marcas de evidencialidade, mas considero somente o sufixo final -ka como tendo função evidencial, marcando a experiência ou sensação do Curupira de ter ficado adormecido. A construção inferencial tem a função alternativa de expressar 'informação inesperada'. Mesmo sendo uma esfera semântica separada da evidencialidade, 'informação inesperada' é expressa em algumas línguas por marcadores de evidencialidade-freqüentemente das categorias de INFERÊNCIA Ou RELATADA-COmo uma de suas extensões semânticas. O uso de tais categorias de evidência externa para expressar uma sensação interna do falante mostra perfeitamente a noção de algo que chega de fora, "que é uma novidade para o falante, algo que ainda não foi integrado a sua visão de mundo" (DeLancey, 1997:36). O Curupira do texto expressa exatamente esse tipo de surpresa ao se encontrar acordado depois de tanto tempo adormecido (morto).

Nessa breve descrição, vimos que há várias categorias de evidencialidade em Wanano que, além de expressar um núcleo semântico ligado a uma tipologia de fontes de informação, podem ser utilizadas para marcar outros sentidos ou situações específicas. Alguns desses usos são claramente extensões da semântica básica da categoria, como, por exemplo, o uso de evidenciais visuaIs para falar de fatos gerais. Outros usos expressam sentidos aparentemente fora da esfera estrita da evidencialidade, como é caso da marcação de 'informação inesperada'. Com certeza, descobriremos ainda outros sentidos e outros usos.

\section{CONSIDERAÇÕES FINAIS}

Sem dúvida, há muitos detalhes e questões relacionadas ao estudo da evidencialidade que, por uma questão de espaço, não puderam ser abordados neste trabalho. Mas podemos pelo menos mencionar três pontos que despertam maior atenção.

Em primeiro lugar, desde a década de 80, debate-se o problema da classificação de evidencialidade como uma categoria modal que expressa valores epistêmicos. Alguns autores argumentam que, ao identificar a fonte de informação, o falante indica, automaticamente, graus diferentes de certeza ou de justificação para a sua afirmação (Anderson, 1986; Chafe e Nichols, 1986; Palmer, 1986; Bybee et al., 1994). Implícita nessa visão é a noção de hierarquização das categorias de forma que: evidência direta > evidência indireta, evidência primeira-mão > informação relatada, evidência visual > outros tipos de evidência sensorial (Willett, 1988; Barnes, 1984). Por outro lado, há autores que defendem a 'neutralidade' dos evidenciais com relação a valores epistêmicos, e afirmam que evidência e certeza são campos semânticos distintos (de Haan, 1999; Aikhenvald, 2003; 2004a). Há ainda quem propõe uma reavaliação completa dos conceitos de modalidade epistêmica e do papel dos evidenciais na codificação de proposições (Givón, 1982; 2001), enquanto outros procuram entender a relação entre evidencialidade e valores epistêmicos por via de conceitos como dêixis (Gomes-Imbert, 1999; Floyd, 1999; de Haan, 2001a). No decorrer desse debate, têm surgido novas descrições de línguas, análises do funcionamento e organização de sistemas 
de evidencialidade que mostram que afirmações 'a priori' quanto à semântica de categorias de evidencialidade não são possíveis. Estudos comparativos como os de Chafe e Nichols (1986) e de Aikhenvald (2003; 2004b) nos mostram tendências, mas cada língua revela uma organização própria das relações entre essas categorias e um conjunto de meios utilizados para codificá-las.

No caso do Wanano, o sistema de evidencialidade pertence a um paradigma maior de marcadores de modalidade oracional e há uma relação implícita entre a marcação de evidencialidade e valores epistêmicos. As implicações epistêmicas das categorias VISUAL, NÃO-VISUAL e INFERÊNCIA (todas primeira-mão, externa) estão parcialmente ligadas à natureza dêitica da codificação de evidência/informação: são categorias que indicam o envolvimento ou proximidade direta do falante ao evento, enquanto a categoria de suposição (primeira-mão, interna) indica evidência estabelecida por um contrato conceitual entre falantes que compartilham experiências e uma mesma cultura. Somente a categoria de evidência RELATADA pode ser considerada como isenta de valor epistêmico. É até possível que isso explique a notada preferência pela citação direta da fala dos outros: com a citação direta a 'responsabilidade' epistêmica permanece com o falante original. No mais, em Wanano, a 'dúvida', se presente, é indicada por um morfema próprio (7b) e só ocorre com as categorias evidenciais de INFERÊNCIA e SUPOSIÇÃo; ou seja, é um caso altamente marcado. Podemos concluir, então, que um valor default de 'verdade' é implícito em toda afirmação não-marcada (Frajzyngier, 1985).

Finalizo com duas observações ligadas a questões conceituais e tipológicas. A primeira tem a ver com a relação entre a semântica dos evidenciais e a dicotomia das esferas realis/ irrealis. Tradicionalmente, associa-se a marcação de evidência exclusivamente à esfera de realis (referente a situações/estados correntes ou já concluídos). No entanto, no sistema de evidencialidade de Wanano, observamos certa sobreposição dessas esferas, um território de fronteira semântico ocupado pela categoria de suPosıç̃̃̃o. Precisamos repensar a dicotomia tradicional frente a esse tipo de categoria, que tem um pé na esfera do realis-quando marca afirmações de fatos-e outro na esfera do irrealis-quando marca suposições lógicas.

A segunda observação tem a ver com a ocorrência de marcadores de evidência em orações interrogativas. Em Wanano, como em algumas outras línguas, evidenciais que ocorrem em perguntas indicam as expectativas do falante relativas ao conhecimento do interlocutor, uma extensão atípica da semântica básica de evidenciais (Aikhenvald 2003). Ficam essas e outras questões para investigações futuras e concluímos com a constatação: estamos diante de fenômenos lingüísticos intrigantes que merecem maior atenção, principalmente no que concerne às línguas amazônicas, ainda muito pouco estudados nesse aspecto. Para mim mesma e para os meus colegas pesquisadores dessas línguas coloco o desafio de aprofundarmos os nossos estudos sobre a evidencialidade. Faço também um convite, de compartilharmos as nossas descobertas e desenvolvermos estudos comparativos, pois certamente temos muito a contribuir para o entendimento dessa fascinante categoria gramatical. 


\section{REFERÊNCIASBIBLIOGRÁFICAS}

AIKHENVALD, Alexandra Y. \& DIXON, Robert M. W. (1998). Evidentials and Areal Typology: a Case Study from Amazonia. Language Sciences 20:241-257.

AIKHENVALD, Alexandra Y. (2003). Evidentiality in Typological Perspective. In Alexandra Y. Aikhenvald \& Robert M. W. Dixon (eds.). Studies in Evidentiality, pp. 1-31. Amsterdam/Philadelphia: John Benjamins.

.( 2004a). Evidentiality: Problems and Challenges. In Piet van Sterkenburg (ed.). Linguistics Today: facing a greater challenge, pp. 1-29. Amsterdam: John Benjamins. .(2004b). Evidentiality. Oxford: Oxford University Press.

ANDERSON, Lloyd B. (1986). Evidentials, Paths of Change, and Mental Maps: Typologically Regular Asymmetries. In Wallace Chafe \& Johanna Nichols (eds.). Evidentiality: The Linguistic Coding of Epistemology, pp. 273-312. Norwood, NJ: Ablex Publishing Corporation.

BARNES, Janet. (1984). Evidentials in the Tuyuca Verb. International Journal of American Linguistics 50: 255-271.

BYBEE, Joan, PERKINS, Revere, \& PAGLIUCA, William. (1994). The Evolution of Grammar: Tense, Aspect, and Modality in the Languages of the World. Chicago: University of Chicago Press.

CHAFE, Wallace, \& NICHOLS, Johanna (eds.) (1986). Evidentiality: The Linguistic Coding of Epistemology. Vol. 20 in the Series Advances in discourse processes. Norwood, NJ: Ablex Publishing Corporation.

De HAAN, Ferdinand. (1999). Evidentiality and Epistemic Modality: Setting Boundaries. Southwest Journal of Linguistics 18:83-101.

.(2001). The Cognitive Basis of Visual Evidentials. In Alan Cienki, Barbara J. Luka \& Michael B. Smith (eds.). Conceptual and Discourse Factors in Linguistic Structure, pp. 91-106. Stanford: CSLI Publications.

DELANCEY, Scott. (1997). Mirativity: The Grammatical Marking of Unexpected Information. Linguistic Typology 1:33-52.

EPPS, Patience. (2005). Areal Diffusion and the Development of Evidentiality: Evidence from Hup. Studies in Language 29:617-649.

FERGUSON, Judith, HOLLINGER, Cari, CRISWELL, Linda, \& MORSE, Nancy L. (2000). El Cubeu. In Maria Stella González de Pérez \& Maria Luisa Rodríguez de Montes (eds.). Lenguas Indígenas de Colombia: Una Visión Descriptiva, pp. 357-370. Santafé de Bogotá: Instituto Caro y Cuervo.

FLOYD, Rick. (1999). The Structure of Evidential Categories in Wanka Quechua. Dallas: Summer Institute of Linguistics/University of Texas at Arlington.

FRAJZYNGIER, Zygmunt. (1985). Truth and the Indicative Sentence. Studies in Language 9:243-254.

GIVÓN, Talmy. (1982). Evidentiality and Epistemic Space. Studies in Language 6:23-49. .(2001). Syntax. An Introduction. Vol. 1. Amsterdam/Philadelphia: John Benjamins.

GOMEZ-IMBERT, Elsa. (1999). Les Catégories de Base de la Conjugaison dans Deux Langues Tukano Orientales. Ms.

.(sous presse) La Vue ou L'ouie: la modalité cognitive des langues Tukano Orientales. In S. Guentchéva \& J. Landaburu (éds.). L'e éoncé médiatisé. vol II, Louvain: Peeters. 
JONES, Wendel, \& JONES, Paula. (1991). Barasano Syntax. Studies in the Languages of Colombia 2. Dallas: Summer Institute of Linguistics.

LANDABURU, Jon. (2005). Expresión Gramatical de lo Epistémico en Algunas Lenguas del Norte de Suramérica. Paper presented at II Congress on Indigenous Languages of Latin America CILLA, Austin, Texas.

MALONE, Terrell. (1988). The Origin and Development of Tuyuca Evidentials. International Journal of American Linguistics 54:119-140.

MILLER, Marion. (1999). Desano Grammar. Studies in the Languages of Colombia 6. Arlington: Summer Institute of Linguistics/University of Texas.

MORSE, Nancy L., \& MAXWELL, Michael B. (1999). Cubeo Grammar. Studies in the Languages of Colombia 5. Arlington: Summer Institute of Linguistics/University of Texas.

PALMER, Frank. R. (1986). Mood and Modality. Cambridge: Cambridge University Press.

RAMIREZ, Henrique. (1997). A Fala Tukano dos Ye'pâ-Masa, Tomo 1 Gramática. Vol. 1. Manaus: CEDEM.

STENZEL, Kristine. (2004). A Reference Grammar of Wanano, Tese Ph.D., University of Colorado.

WALTZ, Nathan, \& WALTZ, Carolyn. (1997). El Agua, La Roca y El Humo: Estudios Sobre la Cultura Wanana del Vaupés. Santafé de Bogotá: Instituto Lingüístico de Verano.

_..(2000). El Wanano. In Maria Stella González de Pérez \& Maria Luisa Rodríguez de Montes (eds.). Lenguas Indígenas de Colombia, Una Visión Descriptiva, pp. 453-467. Santafé de Bogotá: Instituto Caro y Cuervo.

WILLETT, Thomas. (1988). A Cross-Linguistic Survey of the Grammaticalization of Evidentiality. Studies in Language 12:51-97.

\begin{tabular}{llll} 
& \multicolumn{2}{c}{ Abreviaturas } & \\
$1 / 2 / 3$ & $1^{\mathrm{a}} / 2^{\mathrm{a}} / 3^{\mathrm{a}}$ pessoa & INFER & inferência \\
ADV & adverbializador & INST & instrumento \\
AFF & afetado & INT & interrogativo \\
ANAF & anafórico & INTENS & intensificador \\
AUMEN & aumentativo & INTENT & intenção \\
CLS & classificador & LOC & locativo \\
COM & comitativo & MASC & masculino \\
COMPL & completivo & MOV & movimento \\
CONTR & contrastivo & NÃO.VIS & não-visual \\
COP & cópula & NEG & negativo
\end{tabular}




\begin{tabular}{|c|c|c|c|}
\hline DEF & definido & $\mathrm{NOM}$ & nominalizador \\
\hline DEIC & dêitico & OBJ & objeto \\
\hline DEM & demonstrativo & PERF & perfectivo \\
\hline DEON & deôntico & PL & plural \\
\hline DESID & desiderativo & POSS & possessivo \\
\hline DIFF & difuso & PROG & progressivo \\
\hline DIST & distância & PROX & próximo \\
\hline DUB & dubitativo & REC & recente \\
\hline ENF & ênfase & REF & referencial \\
\hline ESP & específico & REL & relatada \\
\hline EXC & exclusivo & REM & remota \\
\hline FEM & feminino & SG & singular \\
\hline FRUST & frustrativo & SUP & suposição \\
\hline IMPER & imperativo & TMP & temporal \\
\hline IMPERF & imperfectivo & VBZ & verbalizador \\
\hline INC & inclusivo & VIS & visual \\
\hline INCOA & incoativo & & \\
\hline
\end{tabular}

Recebido: 05/06/2006

Aceito: 30/10/2006 